\title{
First-Principles Study of Molecule/Al Interfaces
}

\author{
Rachid Belkada ${ }^{1}$, Yoshiyuki Shirakawa ${ }^{2}$, Masanori Kohyama ${ }^{3}$, \\ Shingo Tanaka ${ }^{3}$ and Jusuke Hidaka ${ }^{2}$ \\ ${ }^{1}$ Japan Science and Technology Agency, Kyoto Prefecture Collaboration of Regional Entities \\ for the Advancement of Technological Excellence, Kyoto 619-0237, Japan \\ ${ }^{2}$ Department of Chemical Engineering and Materials Science, Doshisha University, Kyoto 610-032, Japan \\ ${ }^{3}$ Materials Science Research Group, Research Institute for Ubiquitous Energy Devices, National Institute \\ of Advanced Industrial Science and Technology, Osaka 563-8577, Japan
}

The structural and electronic properties of $\mathrm{C}_{n} \mathrm{H}_{2 n+2} / \mathrm{Al}(110)$ interfaces $(n=5)$ have been studied by first-principles calculations using a plane-wave pseudopotential method coupled with an efficient electronic minimization scheme for large systems. We have examined the stability of vertical and parallel adsorption of a $\mathrm{C}_{5} \mathrm{H}_{12}$ molecule on an $\mathrm{Al}(110)$ surface for various adsorption sites and initial interface distances. It has been found that image interactions between the $\mathrm{C}-\mathrm{H}$ polar bonds and the metal surface with physorption characters dominate the interfacial interactions for both the vertical and parallel cases of the $\mathrm{C}_{5} \mathrm{H}_{12} / \mathrm{Al}$ interfaces. However, for the $\mathrm{C}_{5} \mathrm{H}_{11}$ molecule with a dehydrogenated terminal $\mathrm{C}$ atom, we have observed the formation of a strong interfacial C-Al bond with both covalent and ionic characters. [doi:10.2320/matertrans.47.2701]

(Received August 22, 2006; Accepted October 18, 2006; Published November 15, 2006)

Keywords: organic-metal interface, first-principles calculation, electronic minimization techniques, image interaction model

\section{Introduction}

The important role of organic/metal interfaces is well known; they take place in extremely wide range of applications including electronic and optoelectronic components, coatings, and medical devices, etc. Thus the knowledge on the interfacial structures and properties formed by interactions between organic molecules and metals is of great importance for designing and controlling such interfacial structures and properties for various applications. ${ }^{1)}$

In one of the typical applications of organic/metal systems, there is a problem involving super-fine toner particles used in electro-photographic systems such as printers, copiers, and others. ${ }^{2)}$ The interaction between particles of toner powder is weak and generally regarded as of the van der Waals type. However, the interaction between a toner particle and a metal substrate is important and complex, because of the quite different dielectric constants of the two materials. The toner/metal contact seems to be dominated mainly by electrostatic polarization interactions. Thus, detailed knowledge of the electron density redistributions at organic/metal interfaces should reveal the nature of bonding at such interfaces.

In contrast at organic/metal interfaces, there should also be interface dipoles or chemical bonds just like chemisorption in the case of rather strong interactions with shorter distances. Recently, the formation of an interfacial dipole layer at an organic molecule/metal interface has been attracting enormous attention, since it is directly related to the device performance because it governs the flow of charges across the organic/metal interface. The formation of a large dipole was reported for n-alkane and cyclic alkane adsorption on metal surfaces ${ }^{3)}$ and the detailed mechanism of the dipole formation is still not well understood yet. Two reasons suggested for the induced dipole in the n-alkane/ metal system are the image effect and the push back effect, ${ }^{4)}$ although a controversial view suggests that dehydrogenation of alkane molecules may be responsible for the interface dipole formation.

In this work, we report a study on interfacial electronic structure and bonding mechanisms of short n-alkane molecules $\left(\mathrm{C}_{n} \mathrm{H}_{2 n+2}\right)$ adsorbed on $\mathrm{Al}(110)$ surfaces using the density functional theory (DFT) $)^{5,6)}$ within the local density approximation (LDA). ${ }^{7)}$ Recently, quantitative first-principles calculations of electronic properties of various heterointerfaces have become possible by virtue of advances in the theoretical and computational schemes as shown in Refs. 8-10). We investigate the overall features of the energies of the $\mathrm{C}_{5} \mathrm{H}_{12}$ molecule adsorption on the $\mathrm{Al}(110)$ surface for various adsorption sites, interface distances, and molecular orientations, and examine the bonding nature of the most stable interfacial configurations in detail. We also examine the effect of the dehydrogenation of the terminal $\mathrm{C}$ atom of the alkane molecule.

\section{Computational Method}

We carried out all the calculations using a plane-wave pseudopotential method based on the DFT with the LDA, to obtain total energies and atomic and electronic structures of the n-alkane/Al(110) interfaces. In order to deal with large metallic systems, the electronic ground state is obtained efficiently via the conjugate-gradient technique ${ }^{11)}$ or the residual minimization method-direct inversion of the iterative subspace (RMM-DIIS) algorithm, ${ }^{12,13)}$ combined with efficient charge mixing schemes such as the Kerker ${ }^{14)}$ or Pulay scheme. ${ }^{15)}$ The RMM-DIIS method is suitable for efficient parallel computations with respect to each band. ${ }^{16)}$

Optimized pseudopotentials are used in the separable form ${ }^{17)}$ with the plane-wave cut-off energy of $30 \mathrm{Ry}$, requiring about 22000 plane waves in the present supercell. We use the Troullier-Martins type ${ }^{18)}$ norm-conserved pseudopotentials for C and Al, and use the Hamann's generalizedtype pseudopotential ${ }^{19)}$ for $\mathrm{H}$. Detailed information about these pseudopotentials is listed in our previous study, ${ }^{20)}$ where these could reproduce accurately the structural and 
electronic properties of the $\mathrm{Al}(110)$ surface and $\mathrm{C}_{n} \mathrm{H}_{2 n+2}$ molecule.

In the present calculation, we deal with the $\mathrm{Al}(110)$ ideal surface via a slab consisting of five $\mathrm{Al}(110)$ atomic layers. One $\mathrm{C}_{n} \mathrm{H}_{2 n+2}(n=5)$ molecule is adsorbed on the $(2 \times 2)$ unit cell of the $\mathrm{Al}(110)$ surface. The $(2 \times 2)$ cell includes four $\mathrm{Al}$ atoms in each $\mathrm{Al}(110)$ layer, and the supercell contains 20 $\mathrm{Al}$ atoms and one $\mathrm{C}_{5} \mathrm{H}_{12}$ molecule. The cell size parallel to the $\mathrm{Al}$ surface is set by the Al lattice constant, which was optimized by the total-energy minimization of a fcc $\mathrm{Al}$ crystal using the same theoretical scheme in Ref. 20). The obtained equilibrium lattice constant is $0.395 \mathrm{~nm}$, which is consistent with the recent LDA prediction ${ }^{21)}$ and the experimental value of $0.402 \mathrm{~nm}$ at $0 \mathrm{~K}^{22}$ ) For the $\mathrm{C}_{5} \mathrm{H}_{12}$ molecule, we use the experimental bond lengths and bond angles for the initial configuration of the interface calculations. We deal with the vertical and parallel adsorption, where the molecular axis is vertical and parallel to the $\mathrm{Al}$ surface, respectively. The supercell contains a vacuum region of about 20 a.u. between the surface of the molecular layer and the back $\mathrm{Al}$ surface of the next cell in the $\mathrm{z}$ direction to prevent artefact interactions. The cell size is approximately $16 \times 11 \times 50$ a.u.

Sampling of the Brillouin zone is performed with a uniform mesh of $2 \times 2 k$ points per half of the Brillouin zone. Partial occupancies of the single-particle wave functions are given by the Gaussian broadening scheme ${ }^{23,24)}$ of $0.2 \mathrm{eV}$. The electronic structure is calculated using 58 bands for 92 valence electrons of the $\mathrm{C}_{5} \mathrm{H}_{12} / \mathrm{Al}(110)$ system. The stable configuration is obtained by static relaxation according to the Hellmann-Feynman forces.

As the first step, we have performed the relaxation of the $\mathrm{Al}(110)$ surface, and obtained an optimized geometry of the $\mathrm{Al}(110)$ surface, which has a small inward displacement of the top Al layer as reported in our previous calculations. ${ }^{20)}$ In the interface calculations, the initial $\mathrm{Al}$ atomic positions in the slab are those from the final relaxed positions of the $\mathrm{Al}$ surface.

For the vertical adsorption, we first examine the energy variation for the change of the interface distance on several adsorption sites by rigidly translating the molecule without relaxation, so as to clarify the overall features of the molecule over the $\mathrm{Al}(110)$ surface. Here the interface distance is defined as the distance between the $\mathrm{C}$ and $\mathrm{Al}$ atoms at the interface. Figure 1(a) shows the four possible adsorption sites, corresponding to the energy extremes, which are the ontop site over the surface atom, site A, the long bridging site, site $\mathrm{B}$, the short bridging site, site $\mathrm{C}$, and the fourfold site, site D. For the lowest-energy adsorption site in this rigid examination, we perform atomic relaxation without any constraints to determine the most stable configuration.

For the parallel adsorption, however, we examine only one adsorption site for simplicity. Figure 1(b) shows the configuration, where the molecular axis is parallel to the $\langle 001\rangle$ direction of the $\mathrm{Al}(110)$ surface and the plane made by $-\mathrm{C}-\mathrm{C}$ $\mathrm{C}$ - atoms of the molecule is normal to the $\mathrm{Al}$ surface. In fact, the parallel configuration bears some resemblance to the experimentally observed interface structure cited $\mathrm{in}^{3)}$ of $\mathrm{n}$ alkane deposited on a [100] fcc metal surface. In Fig. 1(b), the central $\mathrm{C}$ atom of the molecule is located just above the

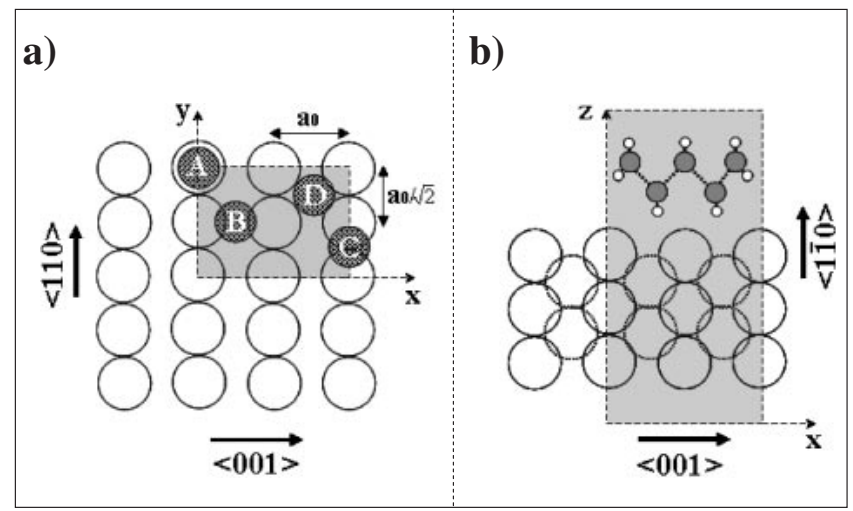

Fig. 1 Schematic description (top view) of the $(2 \times 2)$ cell of the $\mathrm{Al}(110)$ surface including four possible adsorption sites marked from $\mathrm{A}$ to $\mathrm{D}$ of the $\mathrm{C}_{5} \mathrm{H}_{12}$ molecule with its molecular axis is normal to the (x,y) plane (a). Geometry of the parallel orientation (side view) of a $\mathrm{C}_{5} \mathrm{H}_{12}$ molecule adsorption over the $\mathrm{Al}(110)$ surface where the $\mathrm{C}_{5} \mathrm{H}_{12}$ atoms are located over an atomic row of $\mathrm{Al}$ surface atoms (b). The dashed line delimiting the shaded area in (a) and (b) indicate the $(2 \times 2)$ calculation cell in our study.

Table 1 Energy changes (in eV) relative to the minimum energy configuration and its dependence on the interface distances in rigid translations for different vertical adsorption sites demonstrated in Fig. 1(a).

\begin{tabular}{ccccc}
\hline $\begin{array}{c}\text { Interface distances } \\
(\mathrm{nm})\end{array}$ & Site A & Site B & Site C & Site D \\
\hline 0.20 & 2.9818 & 5.2813 & 11.3936 & 9.9196 \\
0.25 & 1.5807 & 2.4822 & 3.48387 & 11.6841 \\
0.30 & 0.5624 & 1.5390 & 1.54451 & 2.2223 \\
0.35 & 0.0 & - & - & - \\
0.40 & 0.5429 & - & - & - \\
0.45 & 0.5748 & - & - & - \\
\hline
\end{tabular}

central $\mathrm{Al}$ atom of the $(2 \times 2)$ cell of the $\mathrm{Al}$ surface. Thus this symmetric configuration has a mirror-plane symmetry with respect to the (001) plane crossing both the central $\mathrm{C}$ atom of the molecule and the central surface $\mathrm{Al}$ atom in the $(2 \times 2)$ unit cell. For this configuration, we first examine the energy variation for the rigid changes in the interface distance without atomic relaxation. The interface distance is defined as the perpendicular distance between the surface $\mathrm{Al}$ atom and the nearest $\mathrm{C}$ atom of the molecule. After this rigid examination, we perform full atomic relaxation to determine the most stable configuration.

\section{Results and Discussion}

For the vertical adsorption, Table 1 lists the results of the total-energy variation in the rigid translation without atomic relaxation for various interface distances and adsorption sites. The energy exhibits a minimum for site A with an interface distance of $0.35 \mathrm{~nm}$ as shown in Table 1 and Fig. 2. This energy is used as the reference in Table 1. In Table 1, sites $\mathrm{B}, \mathrm{C}$ and $\mathrm{D}$ have higher total-energy values than site A for the same interface distance, although the energy might become lower for larger interface distances.

Full atomic relaxation is performed from the initial configurations of site A with several sets of interface distances near to $0.35 \mathrm{~nm}$ in Table 1 to obtain the most 


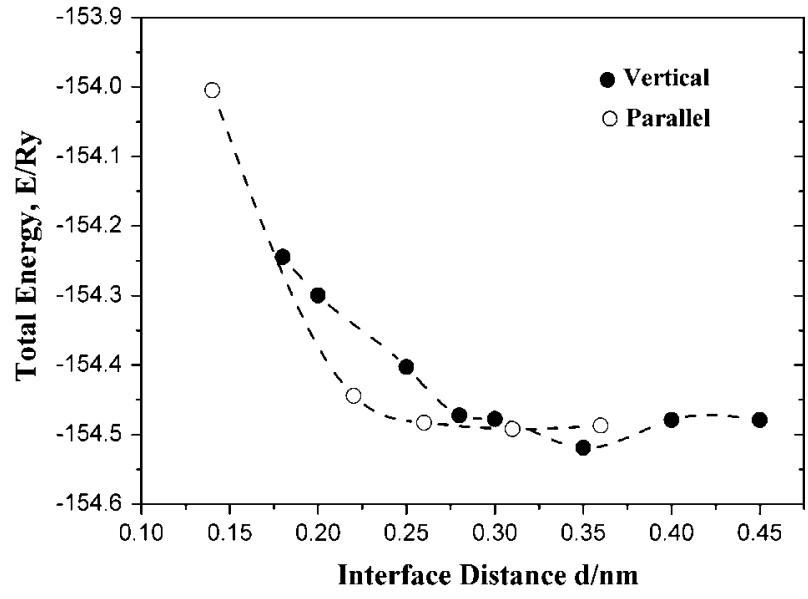

Fig. 2 Energies of the $\mathrm{C}_{5} \mathrm{H}_{12} / \mathrm{Al}(110)$ stable interface configurations obtained in rigid translations for the vertical adsorption corresponding to site A in Fig. 1 (a) and for the parallel adsorption given in Fig. 1(b).

Table 2 Energy variations (in eV) in rigid translation for the parallel adsorption of the molecule as shown in Fig. 1(b). The energy difference values for various interface distances are given with respect to the lower energy adsorption distance.

\begin{tabular}{cl}
\hline $\begin{array}{c}\text { Interface distances } \\
(\mathrm{nm})\end{array}$ & Parallel adsorption \\
\hline 0.14 & 6.6253 \\
0.22 & 0.6459 \\
0.26 & 0.11772 \\
0.31 & 0.0 \\
0.36 & 0.06243 \\
\hline
\end{tabular}

stable configuration efficiently, because the most stable configuration in the rigid translation is not necessarily nearest to the most stable one by the full relaxation. In the obtained most stable configuration for the vertical adsorption, the interface distance is $0.353 \mathrm{~nm}$. The full relaxation leads to a small change of the interface distance, due to a rather large separation and a rather weak interaction. The obtained adsorption energy is of $0.138 \mathrm{eV} /$ molecule, and the adhesive energy is of $0.4 \mathrm{eV} / \mathrm{nm}^{2}$ per unit area, indicating a weak molecular interaction. These values are calculated for the $(2 \times 2)$ cell and represent the energy gain against the sum of the total energies of the relaxed $\mathrm{Al}$ surface and the isolated relaxed molecule, obtained by the same supercell.

For the parallel adsorption, Table 2 and Fig. 2 show the results of the total-energy changes for several interface distances in the rigid translation without relaxation. The energy shows a minimum at an interface distance of $0.31 \mathrm{~nm}$. For a slightly larger distance $(0.36 \mathrm{~nm})$, the energy exhibits a very small increase as shown in Table 2 and Fig. 2. It is interesting that the bottom in the energy-distance curve in Fig. 2 is shallow for the parallel adsorption as compared with that for the vertical adsorption. In Fig. 2, the two curves indicate that the vertical adsorption is a little more stable than the parallel case. However, it should be noted that these curves are the results of rigid translation without relaxation. Indeed, we have found that the parallel adsorption exhibits larger adsorption energy than the vertical one by full atomic relaxation as will be explained below.
The full atomic relaxation for the parallel case is performed from several sets of interface distances near $0.310 \mathrm{~nm}$ in Table 2 to obtain the most stable configuration efficiently. In the obtained most stable configuration, the interface distance is $0.320 \mathrm{~nm}$. In the relaxation procedure, there are no significant changes of the atomic configuration similarly to the vertical case, because of rather weak interactions and a rather large distance. The adsorption energy for the most stable configuration is of $0.322 \mathrm{eV} /$ molecule, and the adhesive energy is of $0.8 \mathrm{eV} / \mathrm{nm}^{2}$ per unit area. It is quite interesting that the adsorption energy and the adhesive energy are twice as large as those of the vertical adsorption. In physorption as in the present cases as analyzed below, the adsorption energy may be proportional to the number of polar $\mathrm{C}-\mathrm{H}$ bonds in contact with the Al surface.

For the parallel case, the distance between the molecules in the neighboring supercells along the $\langle 001\rangle$ axis is not so large, because the supercell size along the $\langle 001\rangle$ axis is rather similar to the molecular size. Of course, we do not deny this might have any effects. However, the adhesion energy in this paper is defined for the molecular system using the same supercell, where the molecules have the same parallel alignment of the interface, and thus the interfacial interaction itself can be adequately evaluated. And in the present configuration, the distance between the neighboring $\mathrm{C}-\mathrm{H}$ bonds of the two molecules is enough large to prevent serious interactions affecting essential results.

To obtain an insight into the electronic properties of the most stable relaxed $\mathrm{C}_{5} \mathrm{H}_{12} / \mathrm{Al}(110)$ interfaces, we have examined the charge redistribution, which is defined as the charge density difference between the interface and the density summation of the separated $\mathrm{Al}$ surface and the isolated molecule having the same geometry as they have in the relaxed interface system. The charge redistribution for the most stable relaxed configuration of the vertical adsorption is shown in Fig. 3, where positive and negative values indicate charge accumulation and depletion, respectively. Substantial charge redistribution is limited at the interface region consisting of the contact $\mathrm{Al}$ atom and the tow or three $\mathrm{CH}_{2}$ groups. There exist clear charge increases near the terminal $\mathrm{H}$ and $\mathrm{C}$ atoms of the molecule in the interface region. The charge redistributions between the $\mathrm{H}$ and $\mathrm{Al}$ atoms seem to be caused by the electrostatic effects of the polar $\mathrm{C}-\mathrm{H}$ bond. However, the magnitude of the charge redistribution is rather small, as compared with the dehydrogenation case as explained later, and there is no electron transfer across the interface as analyzed below, indicating the weak interactions of a physisorption type.

In addition to the above analysis, the amount of charge transfer across the interface can be described by the charge transfer profile, ${ }^{25)} \delta \mathrm{n}(\mathrm{z})=\mathrm{n}(\mathrm{z})-\left(\mathrm{n}_{\mathrm{Als}}(\mathrm{z})+\mathrm{n}_{\text {mol }}(\mathrm{z})\right), \quad$ as shown in Fig. 4, where $n(z)$ is the plane-integrated charge density of the interface at position $\mathrm{z}$, and $\mathrm{n}_{\mathrm{Als}}(\mathrm{z})+\mathrm{n}_{\mathrm{mol}}(\mathrm{z})$ is the superposition of the planar-integrated charge densities of the separate $\mathrm{Al}(110)$ surface and the $\mathrm{C}_{5} \mathrm{H}_{12}$ molecule. In Fig. 4, the interface plane is defined as the position zo, where $\mathrm{n}(\mathrm{zo})$ is minimum between the interfacial $\mathrm{Al}$ and $\mathrm{C}$ atoms. $\delta \mathrm{n}(\mathrm{z})$ corresponds to the planar integration of charge redistributions shown in Fig. 3. It is clear that the charge redistribution is mainly associated with the Al top layer and 

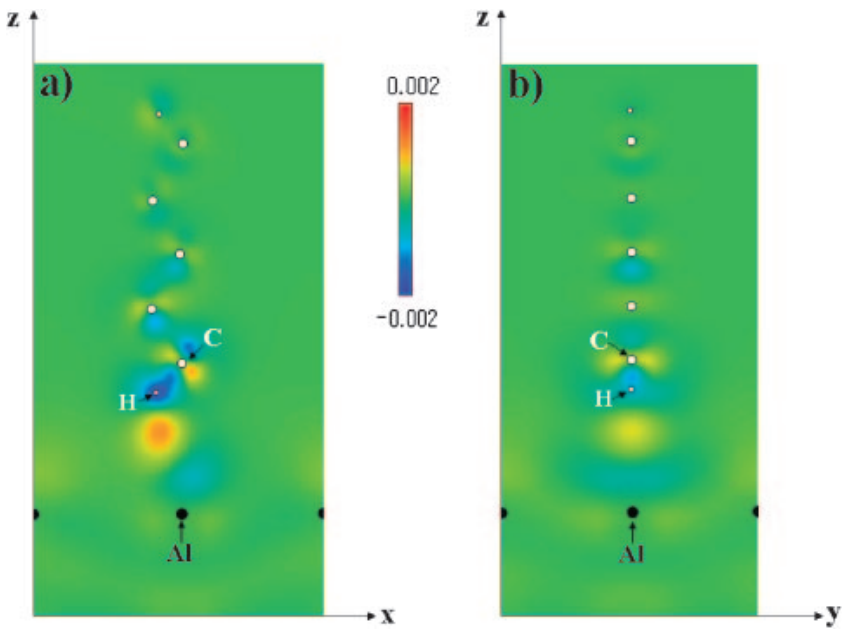

Fig. 3 (Color) Charge redistribution calculated at the most stable interface distance of the relaxed $\mathrm{C}_{5} \mathrm{H}_{12} / \mathrm{Al}(110)$ interface for the vertical adsorption at site A as shown in Fig. 1(a). Slice taken across the middle of the cell in vertical-cut plan parallel to $(\mathrm{x}, \mathrm{z})$ is given in (a) and slice parallel to $(\mathrm{y}, \mathrm{z})$ plane is shown in (b). Positive values represent charge accumulations, whereas negative ones indicate charge depletions.
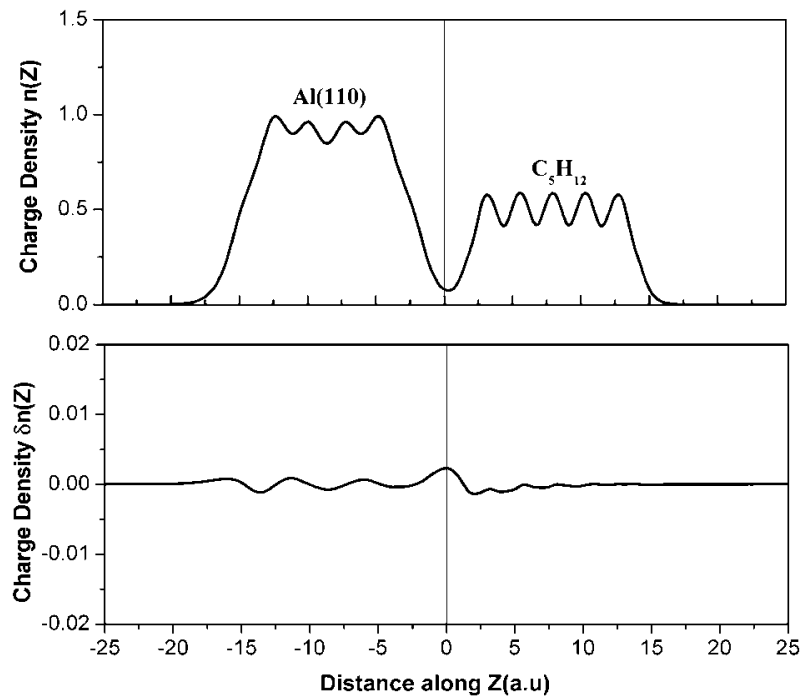

Fig. 4 Plane-integrated charge density profile $n(z)$ and charge-transfer profile $\delta \mathrm{n}(\mathrm{z})$ for the most stable relaxed $\mathrm{C}_{5} \mathrm{H}_{12} / \mathrm{Al}(110)$ interface configuration of the vertical adsorption corresponding to Fig 3. The vertical lines mark the position of the interface plane.

the $\mathrm{CH}_{3}$ group within the interface region, together with rather broad small density oscillations extending into the bulk metal and molecules. The integrated value of $\delta \mathrm{n}(\mathrm{z})$ in Fig. 4 is almost zero, which indicates the physisorption character of the $\mathrm{C}_{n} \mathrm{H}_{2 n+2} / \mathrm{Al}(110)$ system with the vertical configuration, governed by the electrostatic interactions, which bear resemblance to the image charge model. ${ }^{26,27)}$

Figure 5 shows the atomic structure and charge density of the most stable $\mathrm{C}_{5} \mathrm{H}_{12} / \mathrm{Al}(110)$ interface with the parallel adsorption. In Fig. 5(a), it seems that there is no substantial interface bonding charge, but only slight charge overlap between the molecule and the Al surface. In the charge redistribution in Fig. 5(b), there are charge increases between the $\mathrm{H}$ and $\mathrm{Al}$ atoms, which should be caused by the
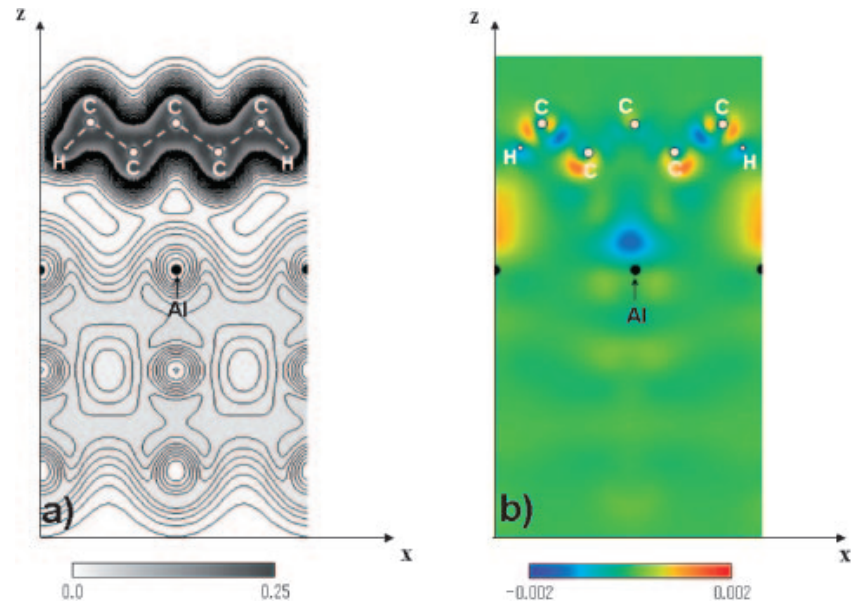

Fig. 5 Most stable atomic structure of the $\mathrm{C}_{5} \mathrm{H}_{12} / \mathrm{Al}(110)$ interface of the parallel adsorption. (001) cross section of the valence charge density distributions (in grey level) and the density contours plotted with an interval spacing of $0.0035 \mathrm{a}^{-\mathrm{u}^{-3}}$ is given in (a). (Color) The corresponding calculated charge redistributions of (a) is indicated in (b).
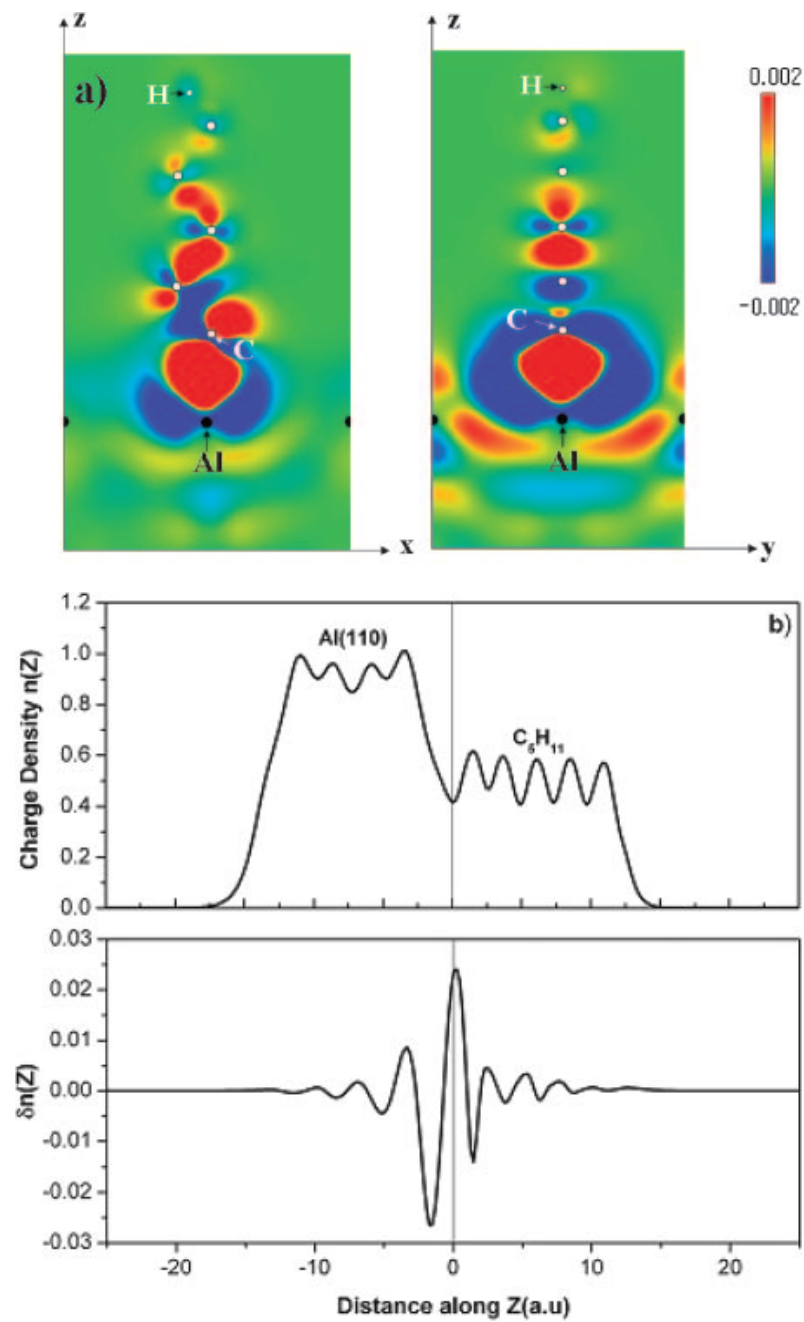

Fig. 6 Final relaxed configuration at a short interface distance of the dehydrogenated $\mathrm{C}_{5} \mathrm{H}_{11}$ molecule for the vertical adsorption at the site $\mathrm{A}$. (Color) Calculated charge redistribution for the $\mathrm{C}_{5} \mathrm{H}_{11} / \mathrm{Al}(110)$ interface (a). The planar-integrated charge density profile $\mathrm{n}(\mathrm{z})$ and the chargetransfer $\delta \mathrm{n}(\mathrm{z})$ profile calculated for the dehydrogenated interface indicated in (a), is given in (b). The interface plane is denoted by vertical lines. 
electrostatic effects of the polar $\mathrm{C}-\mathrm{H}$ bonds, although the charge redistribution between the $\mathrm{C}$ and $\mathrm{Al}$ atoms is quite small and the physical charges transfer between the molecule and the Al surface is also negligible. Therefore, also in the parallel adsorption case, it can be said that the bonding mechanism is the image interaction, where the polar $\mathrm{C}-\mathrm{H}$ bonds electrostatically affect the metallic charge, resulting in the electrostatic interface adhesion.

From the above results of the vertical and parallel adsorptions described in Figs. 3 and 5, the molecule/metal adhesion can be interpreted as due to the presence of highly $\mathrm{C}-\mathrm{H}$ polarized bonds, which induce electrostatic fields near the metal surface affect metallic electrons of Al surface and produce redistributions of electrons to screen the induced electrostatic fields. This feature bears some similarity with the model discussed in Refs. 26) and 27) and described to be an image-charge interaction model, which is likely to occur as a result of the large differences between the dielectric constants of two materials with the molecule $\mathrm{C}_{5} \mathrm{H}_{12}$ having a polarized $\mathrm{C}-\mathrm{H}$ in contact with the metallic charge at the $\mathrm{C}_{5} \mathrm{H}_{12} / \mathrm{Al}$ interface.

To investigate the effect of the dehydrogenation, namely the effect of a $\mathrm{C}$ dangling bond on bonding characteristics, we have examined the vertical adsorption of a $\mathrm{C}_{5} \mathrm{H}_{11}$ molecule by removing the terminal $\mathrm{H}$ atom. For simplicity, we have dealt with an initial short separation distance of $0.18 \mathrm{~nm}$ for site $\mathrm{A}$, and performed the full atomic relaxation of the $\mathrm{C}_{5} \mathrm{H}_{11} / \mathrm{Al}(110)$ system. As shown in Fig. 6(a), the charge redistribution shows a rather strong interaction between the interfacial $\mathrm{C}$ and $\mathrm{Al}$ atoms due to the presence of a $\mathrm{C}$ dangling bond. Significant charge accumulation between the $\mathrm{C}$ and $\mathrm{Al}$ atoms indicates the formation of a covalent $\mathrm{C}-\mathrm{Al}$ bond at the interface. Fig. 6(b) clearly shows that the C-Al bond has polarized charge density where the electrons are pulled strongly from the $\mathrm{Al}$ atom toward the $\mathrm{C}$ atom, due to the difference in the electro-negativity between $\mathrm{C}$ and $\mathrm{Al}$, as observed for $\mathrm{Al}-\mathrm{C}$ interfacial bonds in the $\mathrm{C}$-terminated $\mathrm{SiC}(110) / \mathrm{Al}$ interface. ${ }^{8)}$ It is interesting that the amount of the charge transfer across the interface obtained in Fig. 6(b) from the integration of $\delta \mathrm{n}(\mathrm{z})$ is not very large, and less than $0.1 e$ from $\mathrm{Al}$ toward $\mathrm{C}$. The calculated adsorption energy is of $2.69 \mathrm{eV} /$ molecule, corresponding to the adhesive energy of $6.1 \mathrm{eV} / \mathrm{nm}^{2}$ per unit area, which is 15 times larger than the vertical $\mathrm{C}_{5} \mathrm{H}_{12} / \mathrm{Al}(110)$ system without dehydrogenation. It is clear that the dehydrogenated $\mathrm{C}_{5} \mathrm{H}_{11} / \mathrm{Al}(110)$ system has a very strong $\mathrm{C}-\mathrm{Al}$ bond with both covalent and ionic characters, similarly to the interfacial bonds at the $\mathrm{C}$ terminated $\mathrm{SiC} / \mathrm{Al}$ interface. It is interesting that the present relaxed $\mathrm{C}$-Al bond length $(0.19 \mathrm{~nm})$ is rather similar to that of the $\mathrm{Al}-\mathrm{C}$ bond at the $\mathrm{SiC} / \mathrm{Al}$ interface. ${ }^{8)}$

\section{Conclusion}

The bonding mechanism at organic molecule/metal interfaces has been examined using the ab-initio pseudopotential method. We have examined the energy variation of the $\mathrm{C}_{5} \mathrm{H}_{12}$ molecule adsorption on the $\mathrm{Al}(110)$ surface for various adsorption sites and interface distances, and obtained the most stable configurations for the vertical and the parallel adsorptions. In both the cases, rather weak interactions between the molecule and the $\mathrm{Al}$ surface can be explained by the image interactions, where the polar $\mathrm{C}-\mathrm{H}$ bonds electrostatically affects the metallic surface charge, resulting in the electrostatic adhesion. On the other hand, for the $\mathrm{C}_{5} \mathrm{H}_{11}$ molecule with the dehydrogenated terminal $\mathrm{C}$ atom, we have found that a strong $\mathrm{C}-\mathrm{Al}$ bond with both covalent and ionic characters is formed for the vertical adsorption.

\section{Acknowledgement}

The present study is supported by the Kyoto prefecture for collaboration of regional entities for the advancement of technological excellence, Japan Science and Technology Agency (JST) as the Kyoto Fine Particle Technology project. The calculations are performed using the IBM-JS20 e-server of Doshisha University and Keihanna Inc.

\section{REFERENCES}

1) H. Ishii, K. Sugiyama, E. Ito and K. Seki: Adv. Mater. 8 (1999) 605625.

2) M. Yoshida, N. Li, A. Shimosaka, Y. Shirakawa and J. Hidaka: Chem. Eng. Sci. 61 (2006) 2239-2248.

3) Y. Morikawa, H. Ishii and K. Seki: Phys. Rev. B 69 (2004) 041403-1041403-4.

4) H. Ishii and K. Seki: In Conjugated Polymer and Molecular Interfaces, ed. by W. R. Salaneck, K. Seki, A. Kahn and J.-J. Pireaux (2002, Marcel Dekker, Inc., New York) pp. 293-349.

5) P. Hohenberg and W. Kohn: Phys. Rev. 136 (1964) B864-B871.

6) W. Kohn and L. J. Sham: Phys. Rev. 140 (1965) A1133-A1138.

7) J. P. Perdew and A. Zunger: Phys. Rev. B 23 5048-5079.

8) J. Hoekstra and M. Kohyama: Phys. Rev. B 57 (1998) 2334-2341.

9) S. Tanaka and M. Kohyama: Phys. Rev. B 64 (2001) 235308-1235308-12.

10) K. Okazaki, Y. Morikawa, S. Tanaka, K. Tanaka and M. Kohyama: Phys. Rev. B 69 (2004) 235404-1-235404-8.

11) D. M. Bylander, L. Kleinman, and S. Lee: Phys. Rev. B 42 (1990) 1394-1403.

12) G. Kresse and J. Furthmüller: Phys. Rev. B 54 (1996) 11169-1-1118612.

13) P. Pulay: Chem. Phys. Lett. 73 (1980) 393-398.

14) G. P. Kerker: Phys. Rev. B 23 (1981) 3082-3084.

15) P. Pulay: J. Comput. Chem. 3 (1982) 556-560.

16) T. Tamura, G. Lu, R. Yamamoto, M. Kohyama, S. Tanaka and Y. Tateizumi: Model. Simu. Mater. Sci. Eng. 12 (2004) 1307-1312.

17) L. Kleinman and D. M. Bylander: Phys. Rev. Lett. 48 (1982) 14251428.

18) N. Troullier and J. L. Martin: Phys. Rev. B 43 (1991) 1993-2006.

19) D. R. Hamann: Phys. Rev. B 40 (1989) 2980-2987.

20) R. Belkada, Y. Shirakawa, M. Kohyama, J. Hidaka and S. Tanaka: Trans. Mater. Res. Soc. Jap. 31 (2006) 219-222.

21) N. Mazari, D. Vaderbilt, A. De Vita and M. C. Payne: Phys. Rev. Lett. 82 (1999) 3296-3299.

22) G. N. Kamm and G. A. Albers: J. App. Phys. 35 (1964) 327-330.

23) C.-L. Fu and K.-M. Ho: Phys. Rev. B 28 (1983) 5480-5486.

24) R. J. Needs, R. M. Martin and O. H. Nielsen: Phys. Rev. B 33 (1986) 3778-3784.

25) R. Yang, S. Tanaka and M. Kohyama: Philo. Magazine Lett. 84 (2004) 425-434.

26) A. M. Stoneham: Appl. Surf. Sci. 14 (1983) 249-259.

27) D. M. Duffy, J. H. Harding and A. M. Stoneham: Phil. Mag. A 67 (1993) 865-895. 